\title{
Vesicoureteral Reflux
}

\section{CAN PROPHYLACTIC ANTIBIOTICS SAFELY BE DISCONTINUED IN CHILDREN WITH VESICOURETERAL REFLUX?}

\author{
AHMED J. AL-SAYYAD,* JOHN G. PIKE AND MICHAEL P. LEONARD \\ From the Division of Pediatric Urology, Children's Hospital of Eastern Ontario, University of Ottawa, Ottawa, Ontario, Canada
}

\section{ABSTRACT}

Purpose: We review the outcome of stopping prophylactic antibiotics in children with persistent vesicoureteral reflux (VUR).

Materials and Methods: We performed a retrospective chart review of patients with VUR followed off antibiotics during the last 12 years. Selection criteria included children 4 years old or older who were toilet trained, could verbalize and had a normal voiding pattern. Exclusion criteria were posterior urethral valves, ectopic ureter, neurogenic bladder and severe voiding dysfunction. Outcome measures were age at stopping antibiotics, duration on and off antibiotics, grade of reflux at the time of stopping antibiotics, the occurrence of urinary tract infections (UTI) and new renal scarring on followup. All patients had upper tract assessment performed with renal ultrasound.

Results: A total of 67 girls ( $85.9 \%)$ and 11 boys (14.1\%) were included in the analysis. VUR was bilateral in 36 patients $(46.2 \%)$, on the left side in $29(37.2 \%)$ and on the right side in $13(16.7 \%)$. Reflux was grade I in $16.7 \%$ of the cases, II in $75.6 \%$ and III in $7.7 \%$. Mean patient age when taken off antibiotics was 5.74 years. The period on prophylactic antibiotics ranged from 0 to 84 months (mean 26.2) and the period off antibiotics ranged from 5 to 138 months (37.7). UTI developed in 9 girls (11.5\%), of whom 8 had cystitis (10.2\%) and 1 had clinically presumptive pyelonephritis (1.3\%). These 9 girls were off antibiotics 5 to 60 months (mean 21.1). None of our patients, including those with UTI, had new renal scarring on followup renal ultrasound.

Conclusions: Discontinuing prophylactic antibiotics in selected school age children is safe practice. The risk of significant upper tract infection is low and the development of new renal scars unlikely. However, we fully acknowledge that renal ultrasound is not the most accurate investigation for detection of renal scars. Therefore, continuing medical surveillance of these children is important.

KEY WORDS: vesico-ureteral reflux, antibiotic prophylaxis

Vesicoureteral reflux (VUR) is commonly seen in pediatric urological practice, and is usually initially managed with antibiotic prophylaxis with the anticipation of spontaneous resolution. Surgical intervention has traditionally been reserved for those children who have febrile breakthrough urinary tract infections (UTI), progression of renal scarring, noncompliance with medical therapy or nonresolution of VUR after prolonged followup. ${ }^{1-4}$ There is a cohort of children with nonresolution of reflux in whom the clinical course has been stable, elimination habits are normal and VUR is of low to mid grade. Such children may be at low risk of UTI and, thus, reflux may not impact on renal outcome. In addition, there exists parental pressure to justify the continued use of antibiotic prophylaxis in stable children with VUR.

The news media are replete with stories of "superbugs" and antimicrobial resistance. A recent publication raised the specter of an increased risk of breast cancer in women who had been treated with antibiotics for various indications. ${ }^{5}$ Although this study did not include children who were treated with antibiotic prophylaxis for VUR, Internet savvy parents in our practice have brought this issue to our attention. The literature contains few publications addressing the

* Correspondence: University of Ottawa, Ottawa, Ontario, Canada (e-mail: aboassil@yahoo.com). safety of discontinuing prophylactic antibiotics for VUR, although it is likely a treatment option that many urologists offer patients. ${ }^{6,7}$ We review the practice of discontinuing antibiotics in select patients with VUR, with particular emphasis on the occurrence of UTI and risk to the upper tracts.

\section{MATERIALS AND METHODS}

We conducted a retrospective chart review of patients with VUR followed off antibiotics at a tertiary pediatric referral center during the last 12 years. The criteria to discontinue antibiotics despite persistent VUR included age 4 years or older, toilet trained, reflux grade less than IV, able to verbalize/communicate and normal voiding pattern or mild voiding dysfunction. Exclusion criteria were posterior urethral valves, ectopic ureter, neurogenic bladder, severe voiding dysfunction and reflux grade greater than IV. In some instances parents had insisted on stopping antibiotic prophylaxis in children younger than 4 years and these patients were included in the analysis. Before discontinuation of antibiotics, treatment options including surgical intervention and continuing prophylactic antibiotics were discussed with the parents. The risks of cessation were outlined before a trial off antibiotics was done. 
Outcome measures included age at stopping antibiotics, duration on and off antibiotics, reflux grade at the time of stopping antibiotics, occurrence of UTI and new renal scarring at followup. Reflux grade was documented on the more severe side in bilateral cases. When prophylactic antibiotics were discontinued reflux grade was determined based on the last cystogram obtained while on prophylactic antibiotics. All patients had routine upper tract assessment performed with interval renal ultrasound, and in most nuclear cystograms were done to follow reflux status. In all patients with UTI a repeat cystogram was performed to document reflux status at that time. Urinary tract infection was characterized as clinically presumptive pyelonephritis when a positive urine culture was associated with fever greater than 38.5C, malaise, flank pain and/or peripheral leukocytosis. Suprapubic discomfort, frequency, urgency, dysuria, wetting and/or temperature less than $38.5 \mathrm{C}$ characterized cystitis. All patients diagnosed with UTI had positive urine cultures (greater than $\left.10^{8} \mathrm{cfu} / \mathrm{l}\right)$. Data were analyzed using a summary statistics package provided by SPSS 10.0 statistics software (SPSS, Inc., Chicago, Illinois).

\section{RESULTS}

A total of 67 girls (85.9\%) and 11 boys (14.1\%) were included in the study. Among the cohort analyzed were 10 patients younger than 4 years whose parents chose to stop prophylactic antibiotics against our advice, including 1 child whose parents refused to use antibiotic prophylaxis from the outset. VUR was bilateral in 36 patients $(46.2 \%)$, on the left side in $29(37.2 \%)$ and on the right side in $13(16.7 \%)$. Reflux was grade I in $16.7 \%$ of the cases, II in $75.6 \%$ and III in $7.7 \%$. All patients were infection-free while on prophylaxis. Patient age when taken off of antibiotics ranged from 1 to 12 years (mean of 5.74). The period on prophylactic antibiotics before discontinuance ranged from 0 to 84 months (mean 26.2), and the period off of antibiotics ranged from 5 to 138 months (37.7).

UTI developed in 9 girls (11.5\%), of whom 8 had cystitis $(10.2 \%)$ and 1 had clinically presumptive pyelonephritis (1.3\%). Reflux was bilateral in 4 of these girls and on the left side in 5 , grade II in 8 , including the patient with clinically presumptive pyelonephritis, and grade I in 1 . Period off antibiotics for these 9 girls ranged from 5 to 60 months (mean 21.1). None of the 10 patients younger than 4 years had a UTI, with a period off antibiotics ranging from 12 to 138 months (mean 49.5). None of our patients, including those with UTI, had new renal scarring detected on serial renal ultrasound. In addition, a dimercapto-succinic acid (DMSA) scan several months after infection in the patient with clinically presumptive pyelonephritis was negative.

Further followup of the UTI cohort revealed that thepatient with clinically presumptive pyelonephritis was put on prophylactic antibiotics for an additional 4 years. She was then subsequently taken off of antibiotics for 3 years with no UTI. Of the patients with cystitis 5 continued off antibiotics with no UTI for 2 to 5 years, 2 patients started on antibiotic prophylaxis again and 1 patient opted for a STING procedure.

\section{DISCUSSION}

Accepted treatment alternatives for vesicoureteral reflux are antibiotic prophylaxis or surgical intervention. Considerations for operative intervention include breakthrough UTI, new renal scars/failure of renal growth, non-compliance with medical management, severe initial grade of reflux (IV to V/V) outside of the infant age group, persistent VUR (more than 3 to 4 years) and VUR associated with congenital abnormalities at the ureterovesical junction. Although the longterm use of antibiotics seems innocuous, the cost, inconvenience, possible side effects and parental concerns may prompt surgical intervention. ${ }^{8}$ Previous studies document that the risk of renal scarring with UTI is greatest in children younger than 1 year, and that patients with febrile UTI before age 4 years have a much greater likelihood of renal scarring than older children. ${ }^{9,10}$ Prompt treatment of pyelonephritis is known to prevent renal scar formation. ${ }^{11,12}$ Thus, the concept of discontinuing prophylactic antibiotics in older children with VUR who are communicative and not greatly predisposed to UTI by dysfunctional voiding habits has a sound footing.

Two previous studies have looked at stopping prophylactic antibiotics in children with VUR. Cooper et al followed 51 patients off antibiotics for a period comparable tothat of our study. ${ }^{6}$ UTI developed in $11.8 \%$ of their patients, a rate equivalent to ours. However in their series 5 of 6 patients with UTI had pyelonephritis, and all patients had grade III VUR. Despite the infections, no new renal scarring was seen on subsequent ultrasound. Thompson et al followed 196 patients off antibiotics of an age group and for a period similar to those of our study. ${ }^{7}$ However, their cohort included more patients with severe grades of reflux (IV, V). The rate of UTI per patient per year was 0.29 on and 0.24 off antibiotics. In fact, patients with higher grades of VUR had fewer UTIs per year off versus on prophylactic antibiotics. Similar to our results, Thompson et al found that UTI after stopping antibiotic prophylaxis was more commonly seen in girls. The upper tract in their study was assessed with DMSA renal scans, which are a more accurate means of detecting renal scarring than renal ultrasound. Interestingly the DMSA scans revealed a new renal scarring rate of $2.6 \%$ on antibiotic prophylaxis versus $3.6 \%$ off prophylaxis. Therefore, this study confirmed the safety of cessation of antibiotic prophylaxis even among selected patients with high grade reflux.

The majority of our patients $(88.5 \%)$ did well off antibiotics without any UTI for an average followup interval of 3 years. Admittedly this outcome reflects selection bias, as patients with major voiding dysfunction and/or severe VUR were not offered this observational option. Even among patients in whom a UTI occurred the vast majority had lower tract infection only. None of these patients had evidence of upper tract changes on sequential renal ultrasound.

Routine followup study protocols for the child with VUR off antibiotic prophylaxis have not been established. After stopping prophylactic antibiotics we usually continue with routine ultrasound surveillance. However, we fully acknowledge that renal ultrasound is not the most accurate way of delineating renal scarring, but it is difficult to convince a family that their child should undergo a renal scan in the setting of a lower UTI with a normal renal ultrasound. In the patient with clinically presumptive pyelonephritis a DMSA renal scan obtained several months after the infection failed to reveal any significant upper tract scarring. Thus, it seems that the renal status of our study cohort was not placed at risk by the practice of discontinuing prophylactic antibiotics. Interestingly, no UTI developed in any of the 10 children younger than 4 years whose parents insisted that prophylactic antibiotics cease. The small number of children in this group does not allow us to make any sweeping conclusions, and we would still advocate antibiotic prophylaxis in this age group, as the risk of renal scarring with infection is significant.

A secondary benefit to patients who are being followed with VUR off antibiotics is the ability to forego regular cystogram studies. If the results of a cystogram are not being used to make a clinical decision, the study may be omitted from the followup protocol. Parents and children find the cystogram to be a stressful and painful study, and the option of discontinuing it is attractive. However, the parents are fully aware that should their child suffer a UTI or should there be any significant upper tract changes at followup, a cystogram would be obtained at that time. What of the pa- 
tients who had UTI on the observational protocol? As evidenced in our experience, a second chance off antibiotics seems reasonable, since the majority did not have recurrent infections. However, the option of surgical intervention should always remain open for those families who wish closure.

The long-term outcome of children with persistent reflux off antibiotics remains unknown, especially for females who will approach their reproductive years with reflux status unknown. Such patients could be offered a repeat cystogram at the time of puberty to document whether VUR is persistent, as this may affect obstetrical management in the future. When discharging patients from care we inform them that a febrile UTI would be a reason to seek further urological care.

\section{CONCLUSIONS}

Discontinuing prophylactic antibiotics in select schoolaged children with persistent VUR is safe practice. The risk of significant upper tract infection is low, and the development of new renal scars is unlikely. Continuing surveillance of these children by their family doctor and/or urologist is necessary.

\section{REFERENCES}

1. Bailey, R. R.: The management of grades II (nondilating) vesicoureteral reflux and I. J Urol, 148: 1693, 1992

2. Goldraich, N. P. and Goldraich, I. H.: Followup of conservatively treated children with high and low grade vesicoureteral reflux: a prospective study. J Urol, 148: 1688, 1992

3. Belman, A. B.: Vesicoureteral reflux. Pediatr Clin North Am, 44: 1171,1997

4. McLorie, G. A., McKenna, P. H., Jumper, B. M., Churchill, B. M., Gilmour, R. F. and Khoury, A. E.: High grade vesicoureteral reflux: analysis of observational therapy. J Urol, part 2, 144: 537, 1990

5. Lyman, G. H., Culakova, E. and Griggs, J.: Use of antibiotics and risk of cancer. JAMA, 291: 2700, 2004

6. Cooper, C. S., Chung, B. I., Kirsch, A. J., Canning, D. A. and Snyder, H. M.: The outcome of stopping prophylactic antibiotics in older children with vesicoureteral reflux. J Urol, 163: 269,2000

7. Thompson, R. H., Chen, J. J., Pugach, J., Nasser, S. and Steinhardt, G. F.: Cessation of prophylactic antibiotics for managing persistent vesicoureteral reflux. J Urol, 166: 1465, 2001

8. Elder, J. S., Snyder, H. M., Peters, C., Arant, B., Hawtrey, C. E., Hurwitz, R. S. et al: Variations in practice among urologists and nephrologists treating children with vesicoureteral reflux. J Urol, 148: 714, 1992

9. Winberg, J.: Progressive renal damage from infection with or without reflux. J Urol, 148: 1733, 1992

10. Smellie, J. M., Ransley, P. G., Normand, I. C., Prescod, N. and Edwards, D.: Development of new renal scars. Br Med J, 290: 1957, 1985

11. Smellie, J. M.: Reflections on 30 years of treating children with urinary tract infections. J Urol, 146: 665, 1991

12. Smellie, J. M.: Management of children with severe vesicoureteral reflux. J Urol, 148: 1676, 1992

\section{DISCUSSION}

Dr. Richard Sutherland. How many of your children initially presented with a urinary tract infection versus asymptomatic reflux versus hydronephrosis versus sibling screening?

Dr. Ahmed J. Al-Sayyad. We did not look into that because both groups were started on antibiotics anyway, and I think the rate of resolution for both groups is going to be similar. 